\title{
Dípteros ectoparasitas (Insecta, Diptera) em morcegos (Chiroptera, Mammalia) na Reserva Biológica das Perobas Paraná, Brasil.
}

\author{
Josiane R. R. da Silva ${ }^{1,2}$ \& Henrique Ortêncio Filho ${ }^{1,3}$ \\ 1. Grupo de Estudos em Ecologia de Mamíferos e Educação Ambiental (GEEMEA), Universidade Estadual de Maringá, Av. Reitor Zeferino Vaz, s/n, 87360-000, Goioerê, Paraná, Brasil. \\ (josianerrs@hotmail.com; henfilhobat@gmail.com) \\ 2. Curso de Ciências Biológicas, Universidade Paranaense, Campus Cianorte, Av. Brasil, 1123, 87200-000 Cianorte, Paraná, Brasil. \\ 3. Departamento de Ciências, Universidade Estadual de Maringá, Campus Goioerê.
}

\begin{abstract}
Diversity of ectoparasitic dipterans (Insecta, Diptera) in bats (Chiroptera, Mammalia) from the Perobas Biological Reserve in Paraná, South Brazil. Streblidae and Nycteribiidae are exclusively found in association with bats. The main objective of the present work was to investigate the diversity of bat flies found in bats from the Perobas Biological Reserve, state of Paraná, Brazil. The work was performed in the months of May, June and August, 2008 and February, March and April, 2009. For the capture of bats, 32 mist nets were used, totalizing a capture effort of 43,520 $\mathrm{m}^{2} \mathrm{~h}$. Ectoparasite capture was made manually or through the aid of a forceps. Specimens were conserved in $70 \%$ alcohol and identified with the aid of a stereoscopic microscope. Data was analyzed by means of a non parametric Bootstrap and descriptive statistics. Bat flies species identified were: Aspidoptera falcata Wenzel, 1976, Megistopoda proxima (Séguy, 1926), Megistopoda aranea (Coquillett, 1899), Paratrichobius longicrus (Miranda Ribeiro, 1907), Trichobius tiptoni Wenzel, 1976 and Basilia quadrosae Graciolli \& Moura, 2005. Estimated richness curve indicates tendency to the occurrence of other ectoparasitic species in the conservational unit, since a horizontal asymptote was not achieved. The data obtained is corroborated by the respective data in other Brazilian regions and contribute with information about the group diversity in the Atlantic Forest Biome from the northwest of Paraná.
\end{abstract}

KEYWORDS. Bats, Ectoparasitism, Stational Semidecidual Forest.

RESUMO. Streblidae e Nycteribiidae são encontradas exclusivamente em associação com morcegos. Este trabalho teve como objetivo investigar a diversidade de insetos ectoparasitas encontrados em morcegos da Reserva Biológica das Perobas, Estado do Paraná, Brasil. O trabalho foi realizado nos meses de maio, junho e agosto de 2008 e fevereiro, março e abril de 2009. Para a captura dos morcegos, foram utilizadas 32 redesde-neblina, totalizando esforço de captura de $43.520 \mathrm{~m}^{2}$.h. A coleta de ectoparasitas foi feita manualmente ou com auxílio de pinça reta de ponta fina. Os espécimes foram conservados em álcool $70 \%$ e identificados com auxílio de microscópio estereoscópico. Os dados foram analisados por meio do estimador não paramétrico Bootstrap e estatística descritiva. As espécies de ectoparasitas identificadas foram: Aspidoptera falcata Wenzel, 1976, Megistopoda proxima (Séguy, 1926), Megistopoda aranea (Coquillett, 1899), Paratrichobius longicrus (Miranda Ribeiro, 1907), Trichobius tiptoni Wenzel, 1976 e Basilia quadrosae Graciolli \& Moura, 2005. A curva de riqueza estimada indicou tendência à ocorrência de outras espécies de ectoparasitas na unidade de conservação, haja vista que não foi alcançada a assíntota horizontal. Os dados obtidos corroboram com os verificados em outras regiões do Brasil e contribuem com as informações sobre a diversidade do grupo no bioma Mata Atlântica do noroeste do Paraná.

PALAVRAS-CHAVE. Morcegos, Ectoparasitismo, Floresta Estacional Semidecidual.

Streblidae e Nycteribiidae reúnem dípteros hematófagos ectoparasitos exclusivos de morcegos. São encontrados em todas as regiões biogeográficas, principalmente nos trópicos, parasitando espécies de diversas famílias (Prevedello et al., 2005).

A família Streblidae é representada por 237 espécies em todo o mundo, com a maior diversidade verificada no continente americano: 156 táxons (Dick \& GRACIOLLI, 2008). Nycteribiidae, grupo-irmão de Streblidae, compreende 260 espécies (Graciolli, 2004).

Atualmente no Brasil são conhecidas 68 espécies de Streblidae e 24 de Nycteribiidae, porém os registros estão concentrados principalmente nas regiões Sul e Sudeste (Graciolli et al., 2008). No Estado do Paraná foram registradas 32 espécies de 12 gêneros de Streblidae e nove espécies de um gênero de Nycteribiidae, conferindo ao Paraná o estado com maior riqueza catalogada (Prevedello et al., 2005). Dentre os estudos realizados, podemos destacar o levantamento das espécies paranaenses de Basilia e de seus hospedeiros (Graciolli \& CARVALho, 2001); o inventariamento dos táxons de Nycteribiidae na região de Curitiba (Graciolli, 2004) e em fragmentos de
Floresta com Araucária, também na capital paranaense (Graciolli \& Bianconi, 2007).

Segundo Prevedello et al. (2005), na região noroeste do Paraná praticamente não há trabalhos sobre ectoparasitas de morcegos; destaca-se apenas o estudo realizado no Parque do Cinturão Verde, município de Cianorte (Anderson \& OrTÊNCIO Filho, 2006). Desta forma, o objetivo deste trabalho é investigar a riqueza de espécies de insetos ectoparasitas de morcegos na Reserva Biológica das Perobas, Estado do Paraná, sul do Brasil.

\section{MATERIAL E MÉTODOS}

O trabalho foi realizado na Reserva Biológica das Perobas, uma unidade de conservação federal com 8.716 ha (Fig. 1), localizada a $23^{\circ} 52^{\prime} 52^{\prime}$ 'S, $52^{\circ} 44^{\prime} 08^{\prime \prime} \mathrm{W}$ e a $600 \mathrm{~m}$ de altitude, nos municípios de Tuneiras do Oeste e Cianorte, noroeste do Estado do Paraná, sul do Brasil. A área é caracterizada pelo contato entre a Floresta Estacional Semidecidual Submontana, onde predominam perobas (Aspidosperma spp.), cedros (Cedrella fissilis) e palmitos (Euterpe edulis), e a Floresta Ombrófila Mista, 


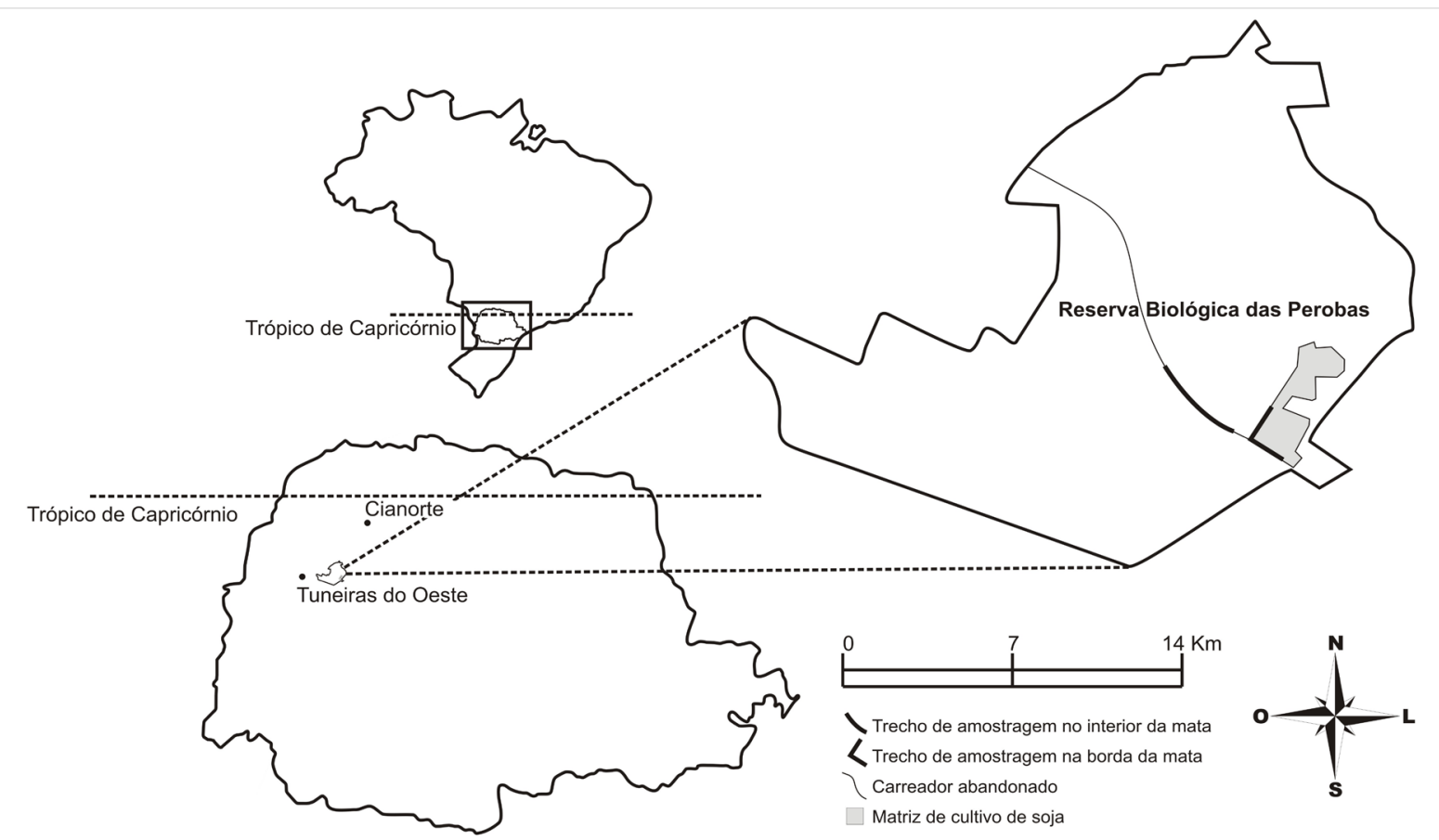

Fig. 1. Localização da Reserva Biológica das Perobas, Paraná, sul do Brasil e os trechos de amostragem.

distinguindo-se pela ocorrência do pinheiro-do-paraná (Araucaria angustifolia) (CASTELla \& BRITEZ, 2004). O clima da região é classificado, segundo Koppen, como subtropical úmido mesotérmico (CFA), com temperaturas médias nos meses mais frios inferiores a $18^{\circ} \mathrm{C}$ e nos períodos mais quentes, acima de $22^{\circ} \mathrm{C}$ (Fuem. Nupélia, 1991).

O estudo foi realizado em uma noite mensal em maio, junho e agosto de 2008 e fevereiro, março e abril de 2009, com início a partir do momento em que a intensidade luminosa foi inferior a 5Lux, por volta de $18 \mathrm{~h}$, e término quando a mesma atingisse valor superior ao anteriormente citado, em torno de $6 \mathrm{~h}$, determinados com o auxílio de luxímetro digital. O tempo médio de amostragem por período noturno foi de 11,3 horas e a amplitude de variação foi igual a 2,5.

Para a captura dos morcegos foram utilizadas, por noite, 32 redes-de-neblina (cada uma com 8,0 $\mathrm{m}$ x 2,5 m) instaladas entre 0,5 e $3,0 \mathrm{~m}$ de altura do solo, totalizando um esforço de captura de $43.520 \mathrm{~m}^{2}$.h (STRaube \& BIANCONI, 2002).

A coleta dos ectoparasitas foi feita com auxílio de pinça reta de ponta fina ou manualmente. Os espécimes foram fixados em álcool $70 \%$, identificados com auxílio de microscópio estereoscópico, seguindo os critérios de Graciolli \& Carvalho (2001) e confirmados pelo Dr. Gustavo Graciolli (Universidade Federal do Mato Grosso do Sul). Os exemplares estão depositados no Laboratório de Pesquisa em Biologia, GEEMEA - Grupos de Estudos em Ecologia de Mamíferos e Educação Ambiental da Universidade Estadual de Maringá, Campus de Goioerê, Paraná.

Para a extrapolação da riqueza de espécies, foi utilizado o software EstimateS (VERSÃo 8.2.0) (COLWELL, 2005), com o método não-paramétrico Bootstrap. Os demais dados foram analisados por meio de estatística descritiva e representados por percentuais.

\section{RESULTADOS}

Foram capturados 170 morcegos das famílias Phyllostomidae, Vespertilionidae e Molossidae, compondo oito gêneros e 13 espécies: Carollia perspicillata (Linnaeus, 1758), Artibeus fimbriatus Gray, 1838, A. lituratus (Olfers, 1818), A. obscurus (Schinz, 1821), A. planirostris (Spix, 1823), Pygoderma bilabiatum (Wagner, 1843), Sturnira lilium (E. Geoffroy, 1810), Molossops neglectus Williams \& Genoways, 1980, Eptesicus brasiliensis (Desmarest, 1819), E. furinalis (d'Orbigny \& Gervais, 1847), Lasiurus blossevillii (Lesson \& Garnot, 1826), Myotis nigricans (Schinz, 1821) e M. ruber (E. Geoffroy, 1806).

Dos morcegos capturados, 26,5\% apresentaram parasitas, sendo $84,5 \%$ Streblidae e $15,5 \%$ Nycteribiidae (Tab. I). Com relação às espécies de Streblidae foram registradas: Aspidoptera falcata Wenzel, 1976, Megistopoda proxima (Séguy, 1926), M. aranea (Coquillett, 1899), Paratrichobius longicrus (Miranda Ribeiro, 1907), Trichobius tiptoni (Wenzel, 1976) e, da família Nycteribiidae, Basilia quadrosae Graciolli \& Moura, 2005; todas as espécies já haviam sido registradas no Estado do Paraná.

Os exemplares coletados de $P$. longicrus estavam sobre o corpo de A. lituratus. Do total amostrado, um percentual de 87,5\% de M. proxima, 75,0\% de $A$. falcata e $77,8 \%$ de $M$. aranea tiveram como hospedeiro $S$. lilium. Trichobius tiptoni ocorreu exclusivamente em 
C. perspicillata, enquanto $B$. quadrosae foi registrada apenas em $M$. nigricans.

A curva de riqueza estimada em relação à curva de espécies observada, baseada no estimador Bootstrap, indicou tendência à ocorrência de outras espécies de ectoparasitas, considerando que não foi alcançada a assíntota horizontal (Fig. 2).

\section{DISCUSSÃO}

O estado paranaense possuía, no final do século XIX, 98\% de Mata Atlântica; atualmente restam apenas 10,52\% (INPE, 2010). Esse bioma abriga a segunda fauna mais rica de mamíferos do Brasil, e no mundo, os morcegos representam, ao menos, $22 \%$ de todos os mamíferos (WiLson \& REEDER, 2005).

De acordo com os registros, no presente estudo os ectoparasitas representaram $16 \%$ das espécies de Streblidae e $9 \%$ das de Nycteribiidae encontradas no Paraná.

$\mathrm{Na}$ Região Neotropical, as comunidades de morcegos são predominantemente compostas por Phyllostomidae (Fenton et al., 1992; Simmons \& Voss, 1998; BiANCONI et al., 2004), existindo uma correlação positiva entre a riqueza de estreblídeos e a de morcegos filostomídeos (Dick \& GetTinger, 2005); este fato também foi verificado no presente estudo, pois, das seis espécies amostradas, cinco pertenciam à Streblidae.

$O$ percentual de morcegos parasitados foi considerado relativamente baixo, representando $26 \%$ do total de morcegos capturados, situação também constatada por GRACIOLLI \& BIANCONI (2007), em Floresta com Araucária no Paraná, sul do Brasil e por Graciolli et al. (2006) em São Paulo, na Estação Ecológica de Caetetus, constituída por floresta semidecídua.

De acordo com as amostragens, $P$. longicrus ocorreu somente em A. lituratus, corroborando com outros estudos em áreas de mesma formação vegetal (Bertola et al., 2005; Anderson \& Ortêncio Filho, 2006), nos quais a referida espécie de morcego foi considerada hospedeira primária do parasita, ou seja, esses organismos passam a maior parte do período de desenvolvimento sobre o corpo do hospedeiro.

Em A. obscurus e A. planirostris os parasitas registrados foram $A$. falcata e $M$. aranea, dados também observados por DiAs et al. (2009) e GUERRERO (1995a,b) na Amazônia, o que nos sugere que essas associações entre ectoparasitas e morcegos são comuns, assim como T. tiptoni em C. perspicillata, também encontrados por BerTOLA et al. (2005).

No presente estudo, foi verificada a ocorrência de $A$. falcata, $M$. proxima e $M$. aranea em $S$. lilium, resultado similar ao obtido por GRACIOLLI \& RUI (2001) e Rui \& Graciolli (2005) em morcegos de fragmentos de Mata Atlântica no Rio Grande do Sul.

Basilia quadrosae foi encontrada pela primeira vez em Mata Atlântica no noroeste do Paraná, perfazendo um único representante da família Nycteribiidae, capturado sobre o corpo de $M$. nigricans. Gracioll \& Moura (2005) assinalaram B. quadrosae sobre E. brasiliensis na Ilha do Cardoso. A ocorrência em $M$. nigricans representa novo registro de hospedeiro e amplia a distribuição da espécie.

Assim como os morcegos Phyllostomidae e os ectoparasitas Streblidae têm uma relação, Nycteribiidae está intimamente associada aos quirópteros Vespertilionidae e a maioria das espécies de Basilia está relacionada ao gênero Myotis (GRACIOLLI \& LinARDI, 2002; GRACIOLLI, 2003, 2004; Bertola et al., 2005). O número reduzido de espécies de Nycteribiidae registrado está associado às baixas taxas de captura de seus hospedeiros, principalmente morcegos Vespertilionidae (Graciolli, 2004).

Além disso, em uma mesma população de morcegos a abundância de ectoparasitas pode ser diferente para o

Tab. I. Espécies de morcegos e seus respectivos ectoparasitas amostrados na Reserva Biológica das Perobas, Paraná, sul do Brasil, nos meses de maio, junho e agosto de 2008 e fevereiro, março e abril de 2009 (N, número de indivíduos capturados; \%MP, frequência relativa de morcegos parasitados; \% PM, frequência relativa de ectoparasitas coletados por hospedeiro).

\begin{tabular}{|c|c|c|c|c|c|}
\hline Espécies de morcegos & $\mathrm{N}$ & $\% \mathrm{MP}$ & Espécies de ectoparasitas & $\mathrm{N}$ & $\% \mathrm{PM}$ \\
\hline \multirow[t]{3}{*}{ Artibeus lituratus } & 99 & 11,8 & Paratricobius longicrus & 10 & 83,4 \\
\hline & & & Megistopoda aranea & 01 & 8,3 \\
\hline & & & Megistopoda proxima & 01 & 8,3 \\
\hline \multirow[t]{3}{*}{ Sturnira lilium } & 33 & 60,6 & Aspidoptera falcata & 06 & 30,0 \\
\hline & & & Megistopoda proxima & 07 & 35,0 \\
\hline & & & Megistopoda aranea & 07 & 35,0 \\
\hline Carollia perspicillata & 05 & 60,0 & Trichobius tiptoni & 03 & 100,0 \\
\hline \multirow[t]{2}{*}{ Artibeus obscurus } & 01 & 100,0 & Aspidoptera falcata & 01 & 50,0 \\
\hline & & & Megistopoda aranea & 01 & 50,0 \\
\hline \multirow[t]{2}{*}{ Artibeus planirostris } & 02 & 100,0 & Aspidoptera falcata & 01 & 50,0 \\
\hline & & & Megistopoda aranea & 01 & 50,0 \\
\hline Myotis nigricans & 14 & 50,0 & Basilia quadrosae & 07 & 100,0 \\
\hline
\end{tabular}




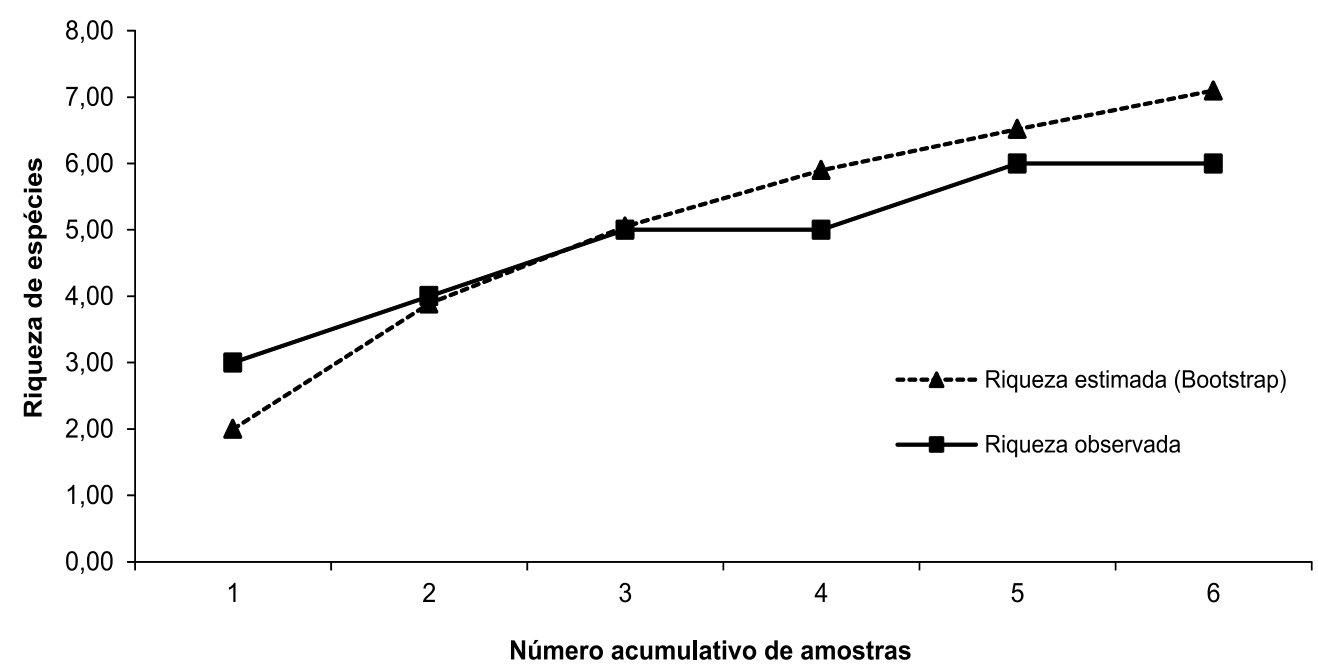

Fig. 2. Riqueza estimada (estimador Bootstrap) e riqueza observada de espécies de ectoparasitas de morcegos da Reserva Biológica das Perobas, Paraná, sul do Brasil, nos meses de maio, junho e agosto de 2008 e fevereiro, março e abril de 2009.

hospedeiro em função da idade, do gênero, das condições reprodutivas e de saúde (Prevedello et al., 2005). Um morcego com alta quantidade de parasitas pode indicar estado de debilitação, refletida pela ausência de autolimpeza, e pode incapacitar ainda mais o hospedeiro (RUI \& Graciolli, 2005).

Emoutrasáreas defloresta estacional semidecidual, nos estados do Rio Grande do Sul (Rui \& Graciolli, 2005) e Paraná (ANDERson \& OrTÊNCIO FiLho, 2006), em área de floresta ombrófila densa do Estado de São Paulo (Bertola et al., 2005) e em área com predomínio de cerrado em Minas Gerais (Komeno \& Linhares, 1999), foram verificadas interações semelhantes entre parasita e hospedeiro se comparadas às observações efetuadas na Reserva Biológica das Perobas; por isso, é relevante a realização de novos estudos em outros locais, para que haja maior compreensão dos ectoparasitas e sua relação com as espécies de morcegos.

De acordo com os dados revelados neste estudo, a maior parte das informações obtidas era esperada por já terem sido descritas na literatura em outras regiões do país, porém, foram constatadas novas associações entre parasitas e hospedeiros. Além disso, os dados sugerem que a riqueza de espécies na área seja superior à constatada no presente estudo.

\section{REFERÊNCIAS BIBLIOGRÁFICAS}

Anderson, R. \& ORTÊNCIO Filho, H. 2006. Dípteros ectoparasitas (Diptera, Streblidae) em filostomídeos (Chiroptera, Mammalia) do Parque Cinturão Verde de Cianorte, Paraná, Brasil e sua incidência ao longo das estações do ano. Chiroptera Neotropical 12(1):238-243.

Bertola, P. B.; Aires, C. C.; Favorito, S. E.; Graciolli, G.; Amaku, M. \& Rосна, R. P. 2005. Bat flies (Diptera: Streblidae, Nycteribiidae) parasitic on bats (Mammalia: Chiroptera) at Parque Estadual da Cantareira, São Paulo, Brazil: parasitism rates and host-parasite associations. Memórias do Instituto Oswaldo Cruz 100(1):25-32.

Bianconi, G. V.; Mikich, S. B. \& Pedro, W. A. 2004. Diversidade de morcegos (Mammalia, Chiroptera) em remanescentes florestais do município de Fênix, noroeste do Paraná, Brasil. Revista Brasileira de Zoologia 21(4):943-954.
Castella, P. R. \& Britez, R. M. 2004. A floresta com araucária no Paraná: conservação e diagnóstico dos remanescentes florestais. Ministério do Meio Ambiente, Brasília. 236p.

Colwell, R. K. 2005. EstimateS 8.2.0: statistical estimation of species richness and shared species from samples. Disponível em: <http://purl.oclc.org/estimates $>$. Acesso: em 26.08.2010.

Dias, P. A.; Santos, C. L. C.; Rodrigues, F. S.; Rosa, L. C.; Lobato, K. S. \& Rebelo, J. M. M. 2009. Espécies de moscas ectoparasitas (Diptera, Hippoboscoidea) de morcegos (Mammalia, Chiroptera) no Estado do Maranhão. Revista Brasileira de Entomologia 53(1):128-133.

Dick, C. W. \& GetTinger, D. 2005. A faunal survey of streblid bat flies (Diptera: Streblidae) associated with bats in Paraguay. Journal of Parasitology 91(2): 1015-1024.

Dick, C. W. \& Graciolli, G. 2008. Checklist of World Streblidae (Diptera: Hippoboscoidea). National Science Foundation. $<$ http://fm1.fieldmuseum.org/aa/Files/cdick/ Streblidae Checklist_18sep08.pdf $>$. Acesso em: 06.06.2010.

Fenton, M. B.; Acharya, L.; Audet, D.; Hickey, M. B. C.; Merriman, C.; Obrist, M. K.; Syme, D. M. \& Adkins, B. 1992. Phyllostomid bats (Chiroptera, Mammalia) as indicators of habitat disruption in the neotropics. Biotropica 24(3):440-446.

Fuem. Nupélia. 1991. Estudos ambientais da planície de inundação do rio Paraná no trecho compreendido entre a foz do rio Paranapanema e o reservatório de Itaipu. Projeto de pesquisa associado ao Curso de Pós-Graduação em Ecologia de Ambientes Aquáticos Continentais. Fuem-Nupélia, Maringá.

Graciolli, G. 2003. Nova espécie de Anatrichobius Wenzel, 1966 (Diptera, Streblidae) do Brasil meridional. Revista Brasileira de Entomologia 47(1):55-58.

2004. Nycteribiidae (Diptera: Hippoboscoidea) no sul do Brasil. Revista Brasileira de Zoologia 21(1):971-985.

Graciolli, G. \& Bianconi, G. V. 2007. Moscas ectoparasitas (Diptera, Streblidae e Nycteribiidae) em morcegos (Mammalia, Chiroptera) em área de Floresta com Araucária no Estado do Paraná, sul do Brasil. Revista Brasileira de Zoologia 24(1):246-249.

Graciolli, G. \& Carvalho, C. J. B. 2001. Moscas ectoparasitas (Diptera: Hippoboscoidea) de morcegos (Mammalia: Chiroptera) do Estado do Paraná. II. Streblidae. Chave pictórica para gêneros e espécies. Revista Brasileira de Zoologia 18(3):907-960.

Graciolli, G. \& Linardi, P. M. 2002. Some Streblidae and Nycteribiidae (Diptera: Hippoboscoidea) from Maracá Island, Roraima, Brazil. Memórias do Instituto Oswaldo Cruz 97(1):139-141.

Graciolli, G. \& Moura, M. O. 2005. Basilia quadrosae sp. nov. (Diptera: Nycteribiidae), member of the ferruginea group, from Southern Brazil. Zootaxa 1087:33-38.

Graciolli, G. \& Rui, A. M. 2001. Streblidae (Diptera, Hippoboscoidea) em morcegos (Chiroptera, Phyllostomidae) no nordeste do Rio Grande do Sul, Brasil. Iheringia, Série Zoologia 90(1):85-92. 
Graciolli, G.; Passos, F. C.; Pedro, W. A. \& Lim, B. K. 2006. Moscas ectoparasitas (Diptera, Streblidae) de morcegos filostomídeos (Mammalia, Chiroptera) na Estação Ecológica dos Caeteus, São Paulo, Brasil. Revista Brasileira de Zoologia 23(1):298-299.

Graciolli, G.; Azevedo, A. A.; Árzua, M.; Barros-Battesti, D. M. \& LinaRDI, P. M. 2008. Artrópodos ectoparasitos de morcegos no Brasil. In: Pacheco, S.; Marques, R. V. \& Esberárd, C. E. L. org. Morcegos do Brasil: Biologia, Sistemática, Ecologia e Conservação. Porto Alegre, Armazém Digital. p. 123-138.

Guerrero, R. 1995a. Catalago de los Streblidae (Diptera: Pupipara) parasitas de murciélagos (Mammalia: Chiroptera) del Nuevo Mundo. III. Los grupos: dugesii, dunni y phyllostomae del genero Trichobius Gervais 1844. Acta Biológica Venezuelica 15(3/4):1 27.

1995b. Catalago de los Streblidae (Diptera: Pupipara) parasitas de murciélagos (Mammalia: Chiroptera) del Nuevo Mundo. V. Trichobiinae com alas reducidas o ausentes y miscelaneos. Boletín de Entomología Venezolana, Nueva Serie 10:135-160.

Instituto Nacional de Pesquisas Espaciais - INPE. 2010 Desflorestamento de Mata Atlântica ocorridos no período de 2008-2010. Disponível em: $<$ http:// www.inpe.br/noticias/noticia. php?Cod_Noticia=2199>. Acesso em: 15.03.2011.

Komeno, C. A. \& Linhares, A. X. 1999. Bat flies parasitic some phyllostomid bats in Southeastern Brazil: parasitism rates and host-parasite relationships. Memórias do Instituto Oswaldo Cruz 94(1):151-156.
Prevedello, J. A.; Graciolli, G. \& Carvalho, C. J. B. 2005. A fauna de dípteros (Streblidae e Nycteribiidae) ectoparasitos de morcegos (Chiroptera) do Estado do Paraná, Brasil: composição, distribuição e áreas prioritárias para novos estudos. Biociências 13(1):193-209

Rui, A. M. \& Graciolli, G. 2005. Moscas ectoparasitas (Diptera, Streblidae) de morcegos (Chiroptera, Phyllostomidae) no sul do Brasil: associações hospedeiros-parasitas e taxas de infestação. Revista Brasileira de Zoologia 22(2):438-445.

Simmons, N. B. \& Voss, R. S. 1998. The mammals of Paracou, French Guiana: a neotropical lowland rainforest fauna, part 1. Bats. Bulletin of the American Museum of Natural History 237(1):1219.

Straube, F. C. \& Bianconi, G. V. 2002. Sobre a grandeza e a unidade utilizada para estimar esforço de captura com utilização de redesde-neblina. Chiroptera Neotropical 8(1-2):150-152.

Wilson, D. E. \& ReEder, D. M. 2005. Mammal Species of the World: a Taxonomic and Geographic Reference, 3 ed. Baltimore, Johns Hopkins University Press. v. 2, 2135p. 